\title{
Anti-ficolin-2 Antibody: Could it be a Predictor of Proliferative Lupus Nephritis in Lupus Patients? Sylvia Talaat Kamal ${ }^{* 1}$, Mohamed Nazmy Farres ${ }^{1}$, Rehab Ali Ibrahim², Ibrahim Sami Ibrahim ${ }^{1}$, Fatma Abdelrahman Ahmed ${ }^{3}$.
}

Departments of ${ }^{1}$ Allergy and Clinical Immunology, Internal Medicine, ${ }^{2}$ Physical Medicine Rheumatology and Rehabilitation, and ${ }^{3}$ Nephrology, Internal Medicine, Faculty of Medicine, Ain Shams University, Cairo, Egypt

*Correspondence author: Sylvia Talaat Kamal,

Email: drsylviatalaat@gmail.com, ORCID: Sylvia Talaat 0000-0003-3235-2644

\begin{abstract}
Background: Nephritis is a challenging domain of systemic lupus erythematosus (SLE). There is a growing need for identification of a non-invasive marker for diagnosing and monitoring nephritis.

Objective: To explore the relevance of using anti-ficolin-2 antibody (Anti-FCN2) as a biomarker for detecting lupus nephritis (LN), and its relation to renal biopsy histopathology and disease activity.

Patients and Methods: Sixty SLE patients were compared to 30 apparently healthy individuals. Thirty of the patients were LN patients (documented by a recent renal biopsy). Full history, examination and laboratory investigations were done. Activity was assessed by SLE disease activity index (SLEDAI) score, and Anti-FCN2 titer was measured by enzyme-linked immunosorbent assay technique (ELISA).

Results: Forty-four of our SLE patients were in disease activity by SLEDAI score. Anti-FCN2 titer was significantly higher among SLE patients compared to control group ( $\mathrm{p}$ value $<0.001$ ). It was also higher among patients with high disease activity compared to those with low disease activity and cutoff value was at $37 \mathrm{ng} / \mathrm{ml}$ (p value is <0.001). AntiFCN2 titer was significantly higher among patients with LN compared to those without LN ( $p$ value is <0.001) with best cutoff value at $72.50 \mathrm{ng} / \mathrm{ml}$. Regarding LN patients, it was significantly higher among patients with proliferative changes than LN patients with non-proliferative changes ( $\mathrm{p}$ value is 0.05 ) with best cutoff value at $155 \mathrm{ng} / \mathrm{ml}$.

Conclusion: Anti-FCN2 shows promising results as a biomarker for lupus disease activity, especially regarding $\mathrm{LN}$ and proliferative changes. Further longitudinal studies on larger samples are needed to confirm.
\end{abstract}

Keywords: Anti-ficolin-2 antibody, Biomarker, Lupus activity, Lupus nephritis, Systemic lupus erythematosus.

\section{INTRODUCTION}

Systemic lupus erythematosus (SLE) is a multisystem autoimmune illness that has various clinical presentations, including cardiac, renal, dermatological, and neurological signs. It is a multifactorial disease in which both adaptive and innate immunity are involved ${ }^{(\mathbf{1}, 2)}$.

One of the life-threatening domains of SLE is lupus nephritis (LN). Rheumatologists encounter a significant challenge in determining the extent of renal damage and monitoring it. Clinical indicators may not necessarily reflect the specific renal pathophysiology, therefore finding new meaningful non-invasive biomarkers is critical nowadays. Furthermore, this may aid in the development of new therapeutic options ${ }^{(3,4)}$.

One of the key mechanisms of LN is the formation of immune complexes (antigen, autoantibody, and complement), and their deposition in renal tissue causes glomerular damage. The classic, alternative, and lectin pathway (LP) all activate the complement system. Normally apoptotic and necrotic cells are cleared to avoid liberation of intracellular autoantigens. This clearance is promoted by some protective molecules such as $\mathrm{C}_{1} \mathrm{q}$, mannose-binding lectin (MBL), ficolins-1 and 2. So, the presence of autoantibodies against those protective molecules might be one of the possible mechanisms of autoimmunity in lupus ${ }^{(3,5,6)}$.

Ficolins are multimeric proteins that possess collagen and fibrinogen-like domains. They are part of innate immunity, as they are structurally similar to MBL (7). They bind to MBL-associated serine proteases and trigger bacterial lysis by activating the complement pathway via LP. They also play an important function in the clearance of apoptotic cells, which helps to maintain tissue homeostasis. In humans, there are three varieties of ficolins: 1, 2, and 3. Ficolin-1 is located on monocytes, granulocytes, and pulmonary cells' surfaces, however ficolins- 2 and 3 are circulating proteins ${ }^{(3,8)}$.

Ficolin -2 (also called L-ficolin) is a triple helical protein which is produced in liver and secreted in the circulation. It is considered one of the main recognition proteins ${ }^{(9)}$.

Low ficolin-2 plasma levels have been linked to an increased risk of LN in patients with SLE, according to previous studies ${ }^{(\mathbf{1 0}, \mathbf{1 1})}$.

Our study aimed to explore the relevance of using antificolin-2 antibody (Anti-FCN2) as a biomarker for lupus disease activity. Also, to elucidate its relationship to various clinical manifestations especially LN and renal biopsy histopathology. 


\section{PATIENTS AND METHODS}

This case control study was conducted on 90 individuals, 60 patients with SLE compared to 30 apparently healthy individuals who were groupmatched with patients regarding age and gender. SLE diagnosis was confirmed by EULAR/ACR criteria 2019 (12). Patients were recruited from Ain Shams University Hospitals, Nephrology and Rheumatology outpatient clinics and inpatient wards between the period of January and September 2020. Patients with infection, congestive heart failure or malignancy were excluded.

SLE patients were further subdivided according to the presence of $\mathrm{LN}$ into 30 patients with $\mathrm{LN}$ and 30 patients without LN. Nephritis was diagnosed by the presence of proteinuria more than $500 \mathrm{mg} \pm$ active sediments in urine, pyuria, or hematuria. Nephritis was confirmed by a recent renal biopsy (within six months of assessment) and further subclassified according to ISN/RPS 2003 Classification ${ }^{(\mathbf{1 3})}$.

Detailed history taking and clinical examination were done. Also, disease activity was assessed by SLE disease activity index (SLEDAI) score. Patients scoring $\geq 6$ were considered in activity ${ }^{(14)}$.

\section{Laboratory tests:}

Five milliliters of venous blood were drawn under complete aseptic precautions: $2 \mathrm{ml}$ in an EDTA test tube to examine complete blood picture using automated cell counter (Beckman Coulter, California 92821, USA), and erythrocyte sedimentation rate (ESR) by the Westergren method (mm/hour). The remaining $3 \mathrm{ml}$ were allowed to clot in a plain test tube, then centrifuged. The obtained serum was used to test the following: Serum creatinine $(\mathrm{mg} / \mathrm{dl})$ by calorimetric method, anti-dsDNA antibody by indirect immune fluorescence, and serum $\mathrm{C}_{3}$ and $\mathrm{C}_{4}$ were measured by nephelometry. Complete urine analysis was examined to detect active urinary sediments (RBCs, WBCs, proteins, or cast) by urine test strip and microscopic examination. Also, quantification of 24 hours urinary protein was done.

\section{Anti-ficolin-2 antibody assay:}

The kit was enzyme-linked immuno-sorbent assay (ELISA), provided by BT laboratory Co., China, Cat Number: ED0330 $\mathrm{Hu}$ ). The plate was pre-coated with human Anti-FCN2 antibody. Anti-FCN2 present in serum was placed and it bound to antibodies coated on the wells. Then biotinylated human Anti-FCN2 antibody bound to Anti-FCN2 in sample. StreptavidinHRP was added and attached to the Biotinylated AntiFCN2 antibody. After incubation, unbound
Streptavidin-HRP was washed away. Finally, Substrate solution was added, and color developed proportionally to the amount of human Anti-FCN2. An acidic stop solution was added, then blue color turned into yellow. Optical density (OD value) of each well was determined immediately using a micro plate reader set to $450 \mathrm{~nm}$. Defining Anti-FCN2 titer was done using the standard curve.

Ethics approval and consent:

The study was approved by the Ethics Committee of the Internal Medicine, Ain Shams University (FWA00017585). Written informed consent for participation in the study was obtained from subjects, after receiving detailed information about the research. This work has been carried out in accordance with The Code of Ethics of the World Medical Association (Declaration of Helsinki) for studies involving humans.

\section{Statistical analysis}

Data were analyzed using IBM@ SPSS $\odot$ Statistics version 25 (IBM $\odot$ Corp., Armonk, NY, USA). Qualitative data were displayed as number and percent. Quantitative data were presented as mean and standard deviation for parametric data, and median and interquartile range (IQR) for non-parametric data. Chisquare test and Fisher exact test were applied to compare categorical variables between different groups. Student t-test and ANOVA were used to compare normally distributed variables. As for non-normally distributed data, Mann-Whitney and Kruskal-Wallis tests were used. Spearman coefficient was applied to correlate between nonparametric quantitative variables. Best cutoff point was detected using Receiver Operating Characteristic curve (ROC). $\mathrm{P}$ value $<0.05$ was considered significant and $<0.01$ was considered highly significant.

\section{RESULTS}

\section{Demographic and Clinical data of the studied individuals:}

This study encompassed 60 patients with SLE. Their characteristics are present in details in table 1. Forty-four patients $(73.3 \%)$ were considered in high disease activity by SLEDAI score.

Thirty of our patients were diagnosed as LN by renal biopsy. Proliferative glomerulonephritis was detected in 18 (60\%) patients of LN group (11 were class III and 7 were class IV). The other 12 patients (non-proliferative) had changes mainly consistent with class II (9 patients). 
Table 1: Clinical and laboratory characteristics of study groups

\begin{tabular}{|c|c|c|c|c|}
\hline & $\begin{array}{c}\text { SLE with LN } \\
(n=30)\end{array}$ & $\begin{array}{c}\text { SLE without LN } \\
(n=30)\end{array}$ & $\begin{array}{c}\text { Control } \\
(\mathbf{n}=30)\end{array}$ & P value \\
\hline Age (years) & $28.07 \pm 5.74$ & $28.13 \pm 5.55$ & $27.77 \pm 6.64$ & $0.97^{\mathrm{a}}$ \\
\hline Female & $28(93.3)$ & $29(96.7)$ & $28(93.3)$ & $1.00^{\mathrm{b}}$ \\
\hline $\begin{array}{l}\text { Duration of disease } \\
\text { (years) }\end{array}$ & $3(1-6)$ & $3(2-4)$ & NA & $0.65^{\mathrm{c}}$ \\
\hline SLEDAI score & $10(8-12)$ & $6(4-8)$ & NA & $<0.001^{\mathrm{c}}$ \\
\hline SLICC/ACR DI score & $1.5(0-1)$ & $1(0-1)$ & NA & $0.15^{\mathrm{c}}$ \\
\hline \multicolumn{5}{|c|}{ Presence of clinical manifestations: } \\
\hline Hypertension & $7(23.3)$ & $5(16.7)$ & NA & $0.52^{\mathrm{d}}$ \\
\hline Joint & $12(40)$ & $21(70)$ & NA & $0.02^{d}$ \\
\hline Neurological & $2(6.7)$ & 0 & NA & $0.49^{\mathrm{b}}$ \\
\hline Cardiological & $1(3.3)$ & $2(6.7)$ & NA & $1^{\mathrm{b}}$ \\
\hline Hematological & $5(16.7)$ & $6(20)$ & NA & $0.74^{\mathrm{d}}$ \\
\hline Cutaneous & $11(36.7)$ & $18(60)$ & NA & $0.07^{\mathrm{d}}$ \\
\hline Laboratory: & & & NA & \\
\hline $\begin{array}{l}24 \text { hours urinary protein } \\
\text { (gm) }\end{array}$ & $3.53 \pm 0.42$ & $0.14 \pm 0.05$ & NA & $<0.001^{\mathrm{e}}$ \\
\hline $\mathrm{Hb}(\mathrm{gm} / \mathrm{dl})$ & $9.99 \pm 1.96$ & $11 \pm 1.07$ & NA & $\mathbf{0 . 0 2}^{\mathrm{e}}$ \\
\hline S. Creatinine $(\mathrm{mg} / \mathrm{dl})$ & $1.7 \pm 0.04$ & $0.72 \pm 0.18$ & NA & $<0.001^{\mathrm{e}}$ \\
\hline $\mathrm{C} 3(\mathrm{mg} / \mathrm{dl})$ & $84.43 \pm 8.44$ & $91.83 \pm 6.29$ & NA & $<0.001^{\mathrm{e}}$ \\
\hline $\mathrm{C} 4(\mathrm{mg} / \mathrm{dl})$ & $13.47 \pm 3.93$ & $19.03 \pm 4.97$ & NA & $\mathbf{0 . 0 0 3}^{\mathrm{e}}$ \\
\hline +ve Anti-ds DNA & $13(43.3)$ & $3(10)$ & NA & ${0.004^{b}}^{b}$ \\
\hline Therapy: & & & NA & \\
\hline MMF & $14(46.7)$ & $1(3.3)$ & NA & $<\mathbf{0 . 0 0 1}^{\mathrm{b}}$ \\
\hline CNI & $8(26.7)$ & $0(0)$ & NA & $\mathbf{0 . 0 1}^{\mathrm{b}}$ \\
\hline AZA & $5(16.7)$ & $15(50)$ & NA & $0.01^{\mathrm{d}}$ \\
\hline Cyclophosphamide & $7(23.3)$ & $1(3.3)$ & NA & $0.05^{b}$ \\
\hline RTX & $1(3.3)$ & $0(0)$ & NA & $1.00^{\mathrm{b}}$ \\
\hline Plasma exchange & $3(10)$ & $0(0)$ & NA & $0.24^{\mathrm{b}}$ \\
\hline
\end{tabular}

Data are presented as mean \pm standard deviation (SD), median (interquartile range (IQR)) and number and percent (\%). SLE: systemic lupus erythematosus, LN: lupus nephritis, SLEDAI: SLE activity index, SLICC/ACR DI score: systemic Lupus International Collaborating Clinics/American College of Rheumatology Damage Index, S: Serum, MMF: Mycophenolate mofetil, CNI: Calcineurin inhibitors, AZA: Azathioprine, RTX: Rituximab.

a One Way ANOVA test, b Fisher Exact test, c Mann Whitney U test, d Chi square test, e Student t test

\section{Anti-ficolin-2 antibody and SLE:}

Patients with SLE showed significantly higher levels of Anti-FCN2 titer: median 72.5 (35-150) ng/ml compared to 6.5 $(5-10) \mathrm{ng} / \mathrm{ml}$ in healthy controls (P value <0.001). At a cutoff level of $19 \mathrm{ng} / \mathrm{mL}$, Anti-FCN2 had a sensitivity of $100 \%$ and a specificity of $96.7 \%$ for differentiating patients with SLE from healthy individuals (AUC $=0.999$ ).

\section{Anti-ficolin-2 antibody and LN:}

Figure 1 shows that Anti-FCN2 was significantly higher among patients with LN 160 (120-270) ng/ml, compared to $35(25-45) \mathrm{ng} / \mathrm{ml}$ in SLE patients without nephritis (P value <0.001). ROC curve illustrated that it had a good discriminative value at the level of $72.50 \mathrm{ng} / \mathrm{ml}$ between both subgroups (AUC $=1.000$, sensitivity: 100\%, specificity: $100 \%)$. 


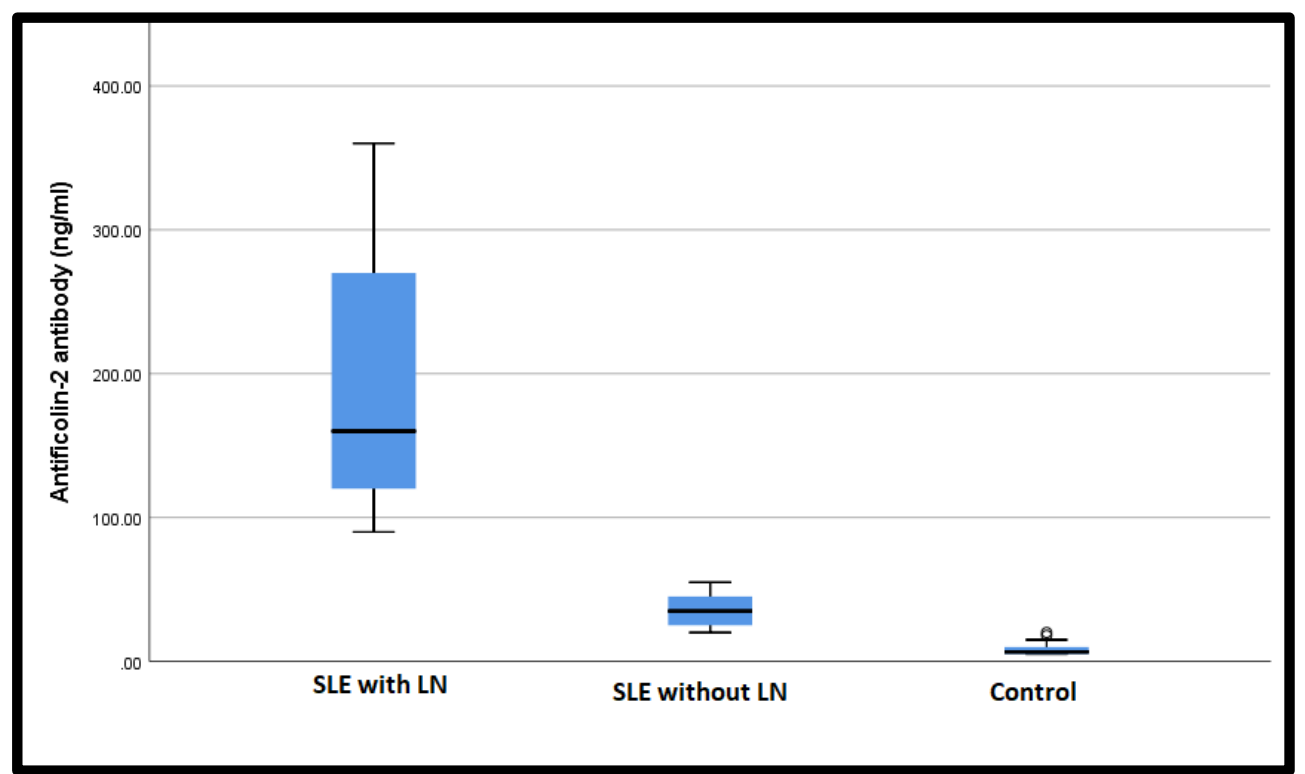

Figure (1) Box plot compares anti-ficolin-2 antibody titer (nglml) between SLE with LN, SLE without LN and control group. $P$ value across the three groups was $<0.001$.

Among patients with $\mathrm{LN}$, Anti-FCN2 antibodies were negatively correlated with platelet count $(\mathrm{r}=-0.416, \mathrm{P}=0.022)$ and $\mathrm{C}_{3}$ level $(\mathrm{r}=-0.374, \mathrm{P}=0.042)$. Another interesting data was a moderate positive correlation between SLEDAI and Anti-FCN2 antibodies $(\mathrm{r}=0.441, \mathrm{P}=0.02)$. Nevertheless, this correlation was not detected among SLE without LN group $(\mathrm{r}=0.191, \mathrm{P}=0.31)$. Additionally, positive anti-dsDNA level in $\mathrm{LN}$ group was associated with significantly higher levels of our marker $160(100-240) \mathrm{ng} / \mathrm{ml}$, compared to $45(32.5-130) \mathrm{ng} / \mathrm{ml}$ among LN patients with negative antidsDNA (P value is 0.01 ).

Regarding the association between Anti-FCN2 antibody titer and renal biopsy, it is clear from figure $2(\mathrm{a}, \mathrm{b})$ that it was significantly higher among patients with proliferative $190(160-280) \mathrm{ng} / \mathrm{ml}$ than patients with non-proliferative renal changes $140(100-180) \mathrm{ng} / \mathrm{ml}$ and $\mathrm{p}$ value was 0.05 . Yet, $\mathrm{P}$ value across the 5 classes was 0.28 . ROC curve showed that $155 \mathrm{ng} / \mathrm{ml}$ was the best cutoff point to discriminate proliferative from non-proliferative changes with sensitivity $77.8 \%$, specificity $75 \%$ with AUC 0.718 .

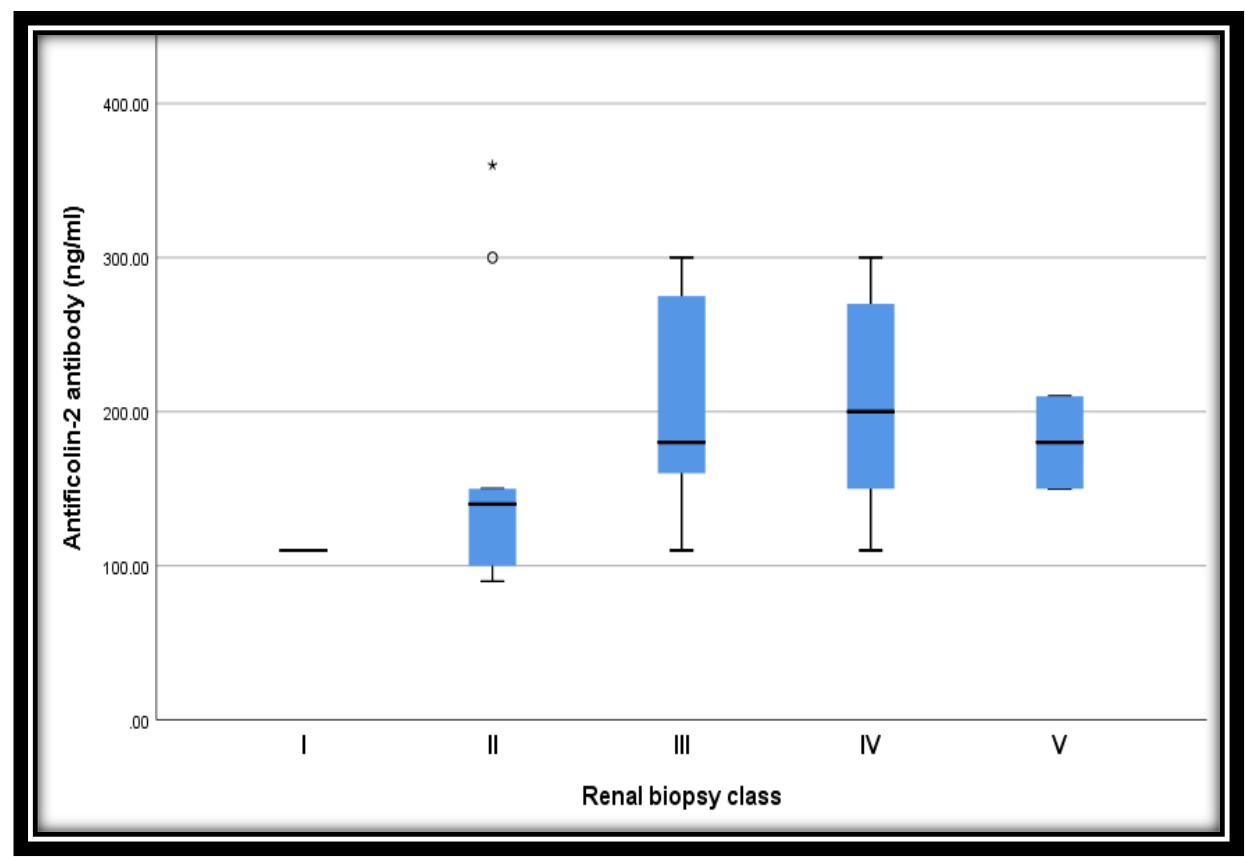

Figure 2 (a) 


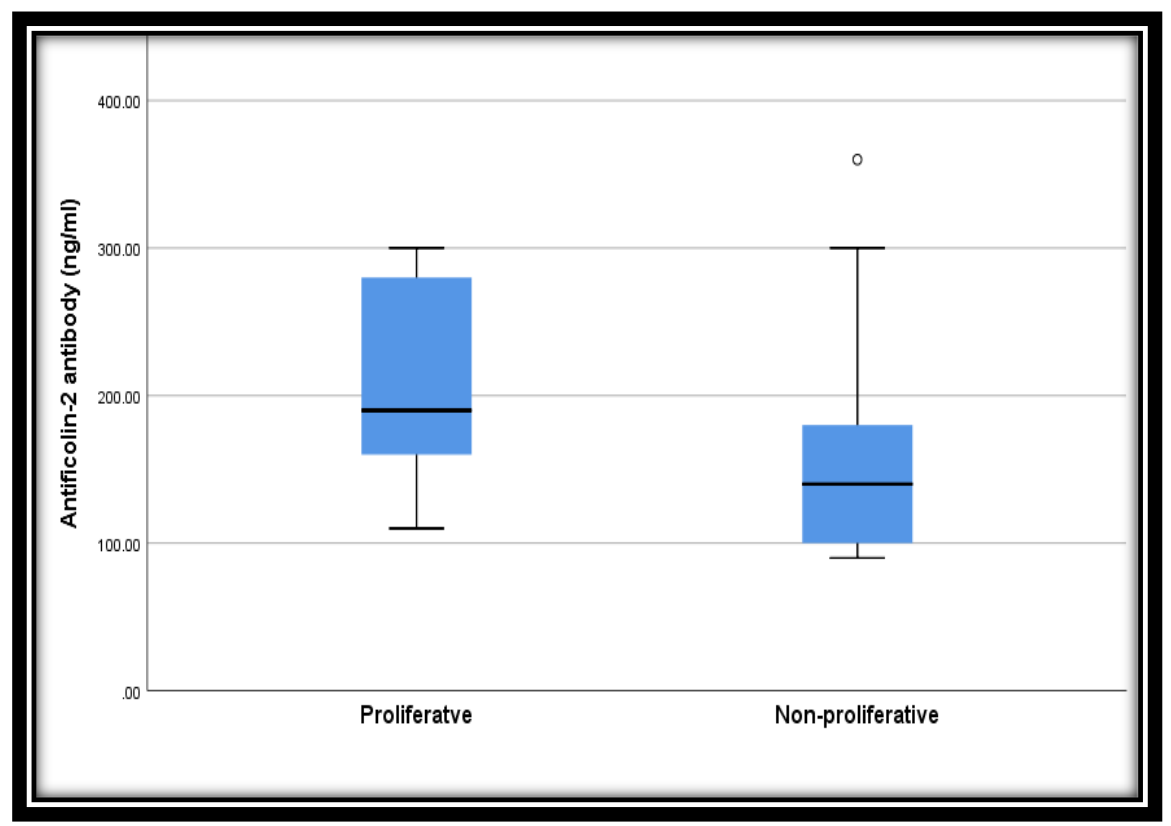

Figure 2(b)

Figures (2): Anti-ficolin-2 antibody level in relation to renal biopsy. Figure (2 a) shows anti-ficolin-2 antibody level in relation to proliferative and non-proliferative $L N(P$ value 0.05$)$. Figure $(2 b)$ shows anti-ficolin-2 antibody level between different renal biopsy classes in patients with lupus nephritis (P value 0.28 ).

Anti-ficolin-2 antibody and disease activity:

Interestingly, on classifying the 60 SLE patients according to disease activity, anti-ficolin-2 antibody titer was also significantly higher among patients with active disease 130 (45-205) ng/ml, compared to 30 (25-40) ng/ml among inactive patients ( $\mathrm{P}$ value is $<0.001$ ). ROC curve had a good discriminative value for diagnosing active SLE at a cutoff level $>37.50 \mathrm{ng} / \mathrm{ml}$ (Sensitivity: $86.4 \%$, Specificity: $75 \%$, AUC $=0.868)$.

Anti-ficolin-2 antibody and other disease manifestations:

Relationship between anti-ficolin-2 antibody levels and the presence of clinical manifestations among active SLE group are demonstrated in table 2. It is apparent that only patients with LN were associated with higher Anti-FCN2 antibody titer. However, patients suffering from arthritis showed significantly lower Anti-FCN2 antibody titer than those without arthritis.

Table (2): Relation between anti-ficolin-2 antibody levels and presence of clinical manifestations among active SLE patients

\begin{tabular}{|c|c|c|c|}
\hline & & Anti-ficolin-2 antibody (ng/ml) & P value \\
\hline \multirow{2}{*}{ Neurological } & Positive & $230(160-300)$ & \multirow[t]{2}{*}{0.17} \\
\hline & Negative & $115(45-200)$ & \\
\hline \multirow{2}{*}{ Cardiological } & Positive & $35(35-300)$ & \multirow[t]{2}{*}{0.46} \\
\hline & Negative & $140(45-200)$ & \\
\hline \multirow{2}{*}{ Hematological } & Positive & $45(40-240)$ & \multirow[t]{2}{*}{0.52} \\
\hline & Negative & $140(45-180)$ & \\
\hline \multirow{2}{*}{ Cutaneous } & Positive & $95(40-210)$ & \multirow[t]{2}{*}{0.40} \\
\hline & Negative & $130.00(90-160)$ & \\
\hline \multirow{2}{*}{ Joint } & Positive & $47.5(40-160)$ & \multirow[t]{2}{*}{$\mathbf{0 . 0 3}$} \\
\hline & Negative & $155(110-240)$ & \\
\hline \multirow{2}{*}{ LN } & Positive & $160(140-275)$ & \multirow[t]{2}{*}{$<0.001$} \\
\hline & Negative & $40(35-45)$ & \\
\hline
\end{tabular}

Data are presented as median (interquartile range (IQR), LN: Lupus nephritis

A multiple linear regression analysis concluded that among all patients with SLE the presence of LN and SLEDAI score were the only independent factors affecting this marker (Table 3). 
Table (3): Multiple linear regression analysis for factors affecting anti-ficolin-2 antibody level among SLE patients

\begin{tabular}{|l|c|c|c|c|c|c|c|}
\hline & \multicolumn{2}{|c|}{$\begin{array}{c}\text { Unstandardized } \\
\text { Coefficients }\end{array}$} & $\begin{array}{c}\text { Standardized } \\
\text { Coefficients }\end{array}$ & \multirow{2}{*}{$\mathbf{t}$} & \multicolumn{2}{|c|}{$\begin{array}{c}\text { P } \\
\text { value }\end{array}$} & \multicolumn{2}{|c|}{$\begin{array}{c}\text { 95.0\% CI } \\
\text { for B }\end{array}$} \\
\cline { 2 - 4 } \cline { 6 - 8 } & $\mathbf{B}$ & $\begin{array}{c}\text { Standard } \\
\text { Error }\end{array}$ & $\mathbf{B e t a}$ & & & $\begin{array}{c}\text { Lower } \\
\text { Bound }\end{array}$ & $\begin{array}{c}\text { Upper } \\
\text { Bound }\end{array}$ \\
\hline LN & 138.207 & 20.273 & 0.672 & 6.817 & $<\mathbf{0 . 0 0 1}$ & 97.563 & 178.851 \\
\hline SLEDAI score & 5.599 & 2.378 & 0.377 & 2.354 & $\mathbf{0 . 0 2 2}$ & 0.831 & 10.367 \\
\hline $\begin{array}{l}\mathbf{2 4} \text { hr urinary } \\
\text { protein (gm) }\end{array}$ & -6.592 & 4.681 & -0.137 & -1.408 & 0.165 & -15.978 & 2.793 \\
\hline $\begin{array}{l}\text { S. Creatinine } \\
\text { (mg/dl) }\end{array}$ & 4.382 & 12.799 & 0.045 & 0.342 & 0.733 & -21.278 & 30.042 \\
\hline $\mathbf{C 3}$ (mg/dl) & 0.126 & 0.328 & 0.077 & 0.385 & 0.702 & -0.531 & 0.784 \\
\hline C4 (mg/dl) & -0.667 & 1.314 & -0.082 & -0.508 & 0.614 & -3.302 & 1.967 \\
\hline
\end{tabular}

LN: lupus nephritis, 95\% CI=95\% confidence interval, hr: Hours, S: Serum

\section{DISCUSSION}

$\mathrm{LN}$ is a common and serious manifestation of SLE, as it is considered one of the leading causes of

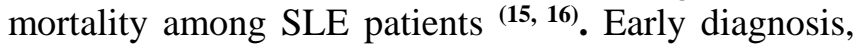
assessment of activity, nephritis classification and hence, appropriate management of LN designates a great challenge to physicians.

Several studies have previously addressed this problem and studied various non-invasive markers yet added a little to clinical practice. This warrants further investigation as advised by EULAR/ERA-EDTA 2019 recommendations ${ }^{(\mathbf{1 7 )} \text {. }}$

In the current study, Anti-FCN2 antibody was evaluated as a marker of lupus activity and particularly LN. We used ELISA as it is a sensitive quantitative method compared to other older qualitative techniques (18).

Anti-FCN2 antibody titers were significantly higher among patients with SLE compared to controls. This is in accordance with the results of Colliard et al. (19) $(\mathrm{P}=0.04)$. The most obvious finding in our study was that patients with LN exhibited significantly higher titers compared to SLE without nephritis. On the other hand, on re-classifying our SLE patients according to lupus disease activity, our marker was much higher in patients with high disease activity compared to those with low activity. This also agreed with the previously mentioned study results Colliard el al. ${ }^{(19)}$. Additionally, anti-FCN2 antibody was positively correlated with SLEDAI in LN group. However, this correlation was not demonstrated in non-LN group. This might show that its correlation with lupus flare is mainly associated with the presence of $\mathrm{LN}$, indicating that anti-FCN2 antibody can be considered as an additional biomarker distinguishing lupus flare from an active $\mathrm{LN}$ flare.

The multiple linear regression analysis applied upon all SLE patients also corroborates the aforementioned results. LN and SLEDAI score were the only factors affecting Anti-FCN2 antibody titer.

SLE does not only have renal manifestations, but may also have joint, cutaneous, cardiac, hematological, and neurological manifestations. In this study, we evaluated various clinical domains relationship to our marker among active patients $(n=44)$. This denoted that anti-FCN2 antibody was only related to $\mathrm{LN}(\mathrm{P}<0.001)$ and this is in agreement with the results of Colliard $\boldsymbol{e} t$ al. ${ }^{(19)}$.

One unanticipated finding was that the titer of anti-FCN2 antibodies was significantly lower in active SLE patients with joint inflammation compared to those without joint inflammation. This agrees with Colliard

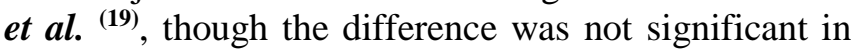
their study. This might be because the majority of patients with arthritis had no nephritis in the current study, and only 12 patients complained of both arthritis and nephritis. This emphasizes that anti-FCN2 antibody titer is mainly related to nephritis activity patients with SLE.

Anti-dsDNA has been observed as an indicator of overall activity in SLE patients and some relate it to renal activity ${ }^{(20)}$. According to our results, anti-FCN2 antibody titer was significantly higher among LN patients with positive anti-dsDNA compared to those with negative results. Besides, complement consumption role as a marker of LN activity is well established in clinical practice. In our study, a significant negative correlation between anti-FCN2 antibody and C3 level was observed. Subsequently, it can be assumed that anti-FCN2 antibody test could be a useful biomarker, added to anti-dsDNA and complement levels for assessment of disease activity in LN patients.

The development of non-invasive markers for the diagnosis and classification of LN, remains a pivotal demand. Hence, a clinically relevant finding in the current study was that anti-FCN2 antibody titer was significantly higher among patients with proliferative compared to those with non-proliferative glomerulonephritis. This is consistent with Colliard $\boldsymbol{e t}$ al. ${ }^{(19)}$ results. However, no significant difference was detected on comparing the five classes. This is assumed to be due to the small sample size especially in classes I and $\mathrm{V}$. This finding has an important implication for 
non-invasive categorization of $\mathrm{LN}$, particularly that repeating a renal biopsy is such a strenuous decision.

\section{CONCLUSION}

These results provide further support for the value of Anti-FCN2 as a marker of lupus activity especially in $\mathrm{LN}$, also as marker of proliferative changes in renal biopsy. Despite these favorable results, questions exist about the exact causal relationship between the presence of anti-ficolin-2 antibodies and LN. Relationship between anti-ficolin -2 antibodies and serum ficolin 2 has to be investigated. Also, further longitudinal studies with larger sample size are recommended.

\section{Authors' contribution:}

Mohamed Nazmy designed the idea and the tools of the study. Fatma Abdelrahman revised step by step the recruitment of patients, and the tools used, Ibrahim Sami collected, analyzed and interpreted the data, Sylvia Talaat proposed the methodology of the study and interpreted the results and Rehab Ali wrote the manuscript. All authors agreed with the results and conclusions of this article. All authors read and approved the final manuscript.

Financial support and sponsorship: Nil.

Conflict of interest: Nil.

\section{REFERENCES}

1. Tsokos G (2011): Systemic lupus erythematosus. N Engl J Med., 365(22):2110-21.

2. Dema B, Charles N (2014): Advances in mechanisms of systemic lupus erythematosus. Discov Med., 17(95):247-55.

3. Dumestre Pérard C, Clavarino G, Colliard S et al. (2018): Antibodies targeting circulating protective molecules in lupus nephritis: Interest as serological biomarkers. Autoimmun Rev., 17(9):890-899.

4. Birmingham D, Merchant M, Waikar S et al. (2017): Biomarkers of lupus nephritis histology and flare: deciphering the relevant amidst the noise. Nephrol Dial Transplant., 32(1): 71-79.

5. Kenyon $\mathrm{K}$, Cole $\mathbf{C}$, Crawford $\mathbf{F}$ et al. (2011): $\mathrm{IgG}$ autoantibodies against deposited $\mathrm{C} 3$ inhibit macrophagemediated apoptotic cell engulfment in systemic autoimmunity. J Immunol., 187(5):2101-11.

6. Fenton K (2015): The effect of cell death in the initiation of lupus nephritis. Clin Exp Immunol., 179(1):11-6.

7. Ichijo H, Hellman U, Wernstedt $C$ et al. (1993): Molecular cloning and characterization of ficolin, a multimeric protein with fibrinogen- and collagen-like domains. J Biol Chem., 268: 14505-13.
8. Rouminina L (2021): The complement system innovative diagnostic and research protocols. Humana; $1^{\text {st }} \quad$ ed., New York, USA. Pp. 121- 132. https://www.amazon.com/-/es/Lubka-TRoumenina/dp/1071610155

9. Matsushita M (2007): The ficolin family: an overview," in Collagen-Related Lectins in Innate Immunity, D. Kilpatrick, Ed., Research Signpost. Pp. 17-31.

10. Watanabe H, Saito R, Asano T et al. (2012): Serum L ficolin levels in patients with systemic lupus erythematosus. Mod Rheumatol., 22:899-902.

11. Tanha N, Pilely K, Faurschou M et al. (2017): Plasma ficolin levels and risk of nephritis in Danish patients with systemic lupus erythematosus. Clin Rheumatol., 36:335341.

12. Aringer M, Costenbader K, Daikh D et al. (2019): 2019 European League Against Rheumatism/American College of Rheumatology Classification Criteria for Systemic Lupus Erythematosus. Arthritis Rheumatol., 71(9): 1400-1412

13. Weening J, D'Agati V, Schwartz M et al. (2004): International Society of Nephrology Working Group on the Classification of Lupus Nephritis; Renal Pathology Society Working Group on the Classification of Lupus Nephritis. The classification of glomerulonephritis in systemic lupus erythematosus revisited. Kidney Int., 65(2):521-30.

14. Bombardier C, Gladman D, Urowitz M et al. (1992): Derivation of SLEDAI. A disease activity index for lupus patients. Arthritis Rheum., 35:630-40.

15. Saxena R, Mahajan T, Mohan C (2011): Lupus nephritis: current update. Arthritis Res Ther., 13(5):24045.

16. Borchers A, Leibushor N, Naguwa $S$ et al. (2012): Lupus nephritis: a critical review. Autoimmun Rev., 12(2):174-94.

17. Fanouriakis A, Kostopoulou M, Cheema $K$ et al. (2020): 2019 Update of the Joint European League against Rheumatism and European Renal AssociationEuropean Dialysis and Transplant Association (EULAR/ERA-EDTA): Recommendations for the management of lupus nephritis. Ann Rheum Dis., 79(6):713-723.

18. Plawecki M, Lheritier E, Clavarino G et al. (2016): Association between the presence of autoantibodies targeting ficolin-3 and active nephritis in patients with systemic lupus erythematosus. PLoS One, 11(9): 160879.

19. Colliard S, Jourde-Chiche $\mathrm{N}$, Clavarino $\mathrm{G}$ et al. (2018): Autoantibodies targeting ficolin-2 in systemic lupus erythematosus patients with active nephritis. Arthritis Care Res (Hoboken), 70: 1263-1268.

20. Isenberg D, Manson J, Ehrenstein M et al. (2007): Fifty years of anti-dsDNA antibodies: are we approaching journey's end? Rheumatol Oxf Engl., 46: 1052-1056. 